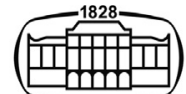

AKADÉMIAI KIADÓ

Acta Chromatographica

33 (2021) 3, 295-301

DOl:

$10.1556 / 1326.2020 .00817$

(c) 2020 The Authors

\section{ORIGINAL RESEARCH} PAPER

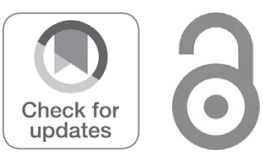

\title{
Study on discrimination of different grades of rapeseed oil based on flavor fingerprint
}

\author{
TONG CHEN ${ }^{1}, \mathrm{CHUNYOU} \mathrm{LIU}^{1}$, BIN CHEN ${ }^{2}$ and \\ YONGCHUN HUANG ${ }^{1 *}$
}

\author{
${ }^{1}$ College of Biological and Chemical Engineering, Guangxi University of Science and Technology, \\ Liuzhou, 545006, PR China \\ ${ }^{2}$ School of Food and Biological Engineering, Jiangsu University, Zhenjiang, 212013, PR China
}

Received: July 14, 2020 • Accepted: July 30, 2020

Published online: August 28, 2020

\begin{abstract}
In this work, Gas chromatograph-Mass Spectrometry (GC-MS) combined with solid phase microextraction technology was used to analyze the difference of volatile organic compounds (VOCs) in rapeseed oil of different grades, and the relationship between changes of VOCs and refining process were also investigated in order to construct a non-linear model, which could realize rapid and accurate discrimination of different grade rapeseed oils. 124 rapeseed oil samples with different grades were collected and analyzed by GC-MS technology and 55 VOCs were identified and selected as variables to characterize the internal quality information of rapeseed oils. Then, principal component analysis (PCA) method was used to extract useful features and reduce data dimensionality, and finally a discriminant model was built using linear discriminant analysis (LDA) algorithm. The correct recognition rate of sample set was close to $94.59 \%$. The results showed that the proposed method is promising in discriminating different grades of vegetable oils. Besides, it provides a theoretical basis for studying the relationship between VOCs composition and vegetable oil quality.
\end{abstract}

\section{KEYWORDS}

GC-MS, rapeseed oil, quality grade, flavor, chemmometrics

\section{INTRODUCTION}

As one of the major cooking oils, rapeseed oil occupies the third position in world's vegetable oil production, after palm oil and soybean oil. Compared to other vegetable oils, such as corn, sesame, sunflower and olive oil, rapeseed oil has a rich content in n-3 fatty acids and n-6/n-3 polyunsaturated fatty acids ratio that is favorable from nutritional point of view, and both of which make it a recommended edible oil [1]. On the other hand, with pleasant flavor and taste [2], rapeseed oil has become a highly acclaimed vegetable oil in the market and is often used as flavor enhancer in Asian countries [3]. Volatile organic compounds (VOCs) are typical secondary characteristic compounds existing in vegetable oils [4], playing a decisive role in the overall flavor of oils and are also one of the key indicators to evaluate oil quality [5]. The main VOCs of vegetable oil are alcohols, aldehydes, ketones and other aromatic substances. Different varieties of vegetable oils contain different types, numbers and contents of flavor components, and even the same variety vegetable oil still retains different VOCs due to difference in natural environment climate, soil quality or processing technology. These are the different flavor components which form the whole flavor and quality of vegetable oils eventually. A critical phase of vegetable oil production chain is the refining process. The free fatty acids of crude oil are effectively removed through refining process to prevent oil rancidity. Meanwhile, other minor components such as pigments, proteins, phospholipids, oxidation products and residue of the solvent used for extraction are also removed in refining process. Therefore, vegetable oils with different refining processes could have different price, 
which would produce a great commercial problem. Therefore, the realization of rapid and accurate determination of quality grade of vegetable oil and the guarantee of the quality consistency of refined oil is of great practical significance and broad application prospect.

At present, many researchers have studied the flavor composition of rapeseed oil. Based on the flavor fingerprint, Gas chromatograph-Mass Spectrometry (GC-MS) technology has been proved suitable for vegetable oil category classification [6], origin identification [7], adulteration detection [8-10], processing flow control $[11,12]$, as well as storage management [13]. These current studies mainly focused on the change of oxidation stability [14], quality indicators such as trace elements and aflatoxins of vegetable oil in the production process [15], and the optimization of process conditions $[16,17]$. However, there are few reports on the changes of flavor components of vegetable oils in the production process, especially refining process. Therefore, in order to realize the rapid discrimination of different grades of vegetable oil based on flavor fingerprint, four grades of rapeseed oil samples were collected to detect flavor composition and to analyze the changes of VOCs in different grades of oil by GC-MS technology combined with solid-phase micro-extraction (SPME). Principal component analysis (PCA) and linear discriminant analysis (LDA) were used to build a non-linear discriminant model, which could provide theoretical basis and technical support for the quality improvement, index formulation and new oil products development.

\section{MATERIALS AND METHODS}

\section{Sample collection}

Four different grades of commercial rapeseed oil samples were collected from Wilmar Global Research and Development Center (Shanghai) and a local supermarket. The raw materials of all oil samples were mainly manufactured in Jiangsu Province, which is the main vegetable oil producing area in China. All refined oil samples were extracted from rapeseeds by traditional solvent extraction method, and the grade differences of product oil were based on the refining progresses, which include degumming, deacidification, bleaching, dewaxing and deodorization technology. The oil samples with different grades were discriminated by Chinese National Standard GB/T 1536-2004. Sample acquisition cycle lasted about six months. The collected samples were stored at $5{ }^{\circ} \mathrm{C}$ in refrigerator and kept away from light in order to obtain enough samples and maintain the inherent quality attribute. Finally, a total of 124 rapeseed oil samples were obtained, among which 31, 34, 26 and 33 samples were labeled grade 1, 2, 3 and 4 respectively.

\section{GC-MS analysis}

All oil samples were analyzed in three times on Agilent HP 6980 GC interfaced to HP 5975 MS using quadrupole mass analyzer. The specific detection conditions are as follows.

\section{(1) Sample preparation}

Firstly, $2 \mathrm{~mL}$ volume of each oil sample was measured by Pipette and was transformed into $10 \mathrm{~mL}$ headspace injection vial, which was sealed with Teflon; Secondly, the oil sample was placed in the thermostatic equipment (water bath), which was equipped with a magnetic stirrer; Thirdly, the sample was balanced for $20 \mathrm{~min}$ in water bath at $60{ }^{\circ} \mathrm{C}$; Finally, the SPME instrument was inserted into the headspace bottle to adsorb the volatile organic compounds from the rapeseed oil, and the extraction fiber was placed at $1 \mathrm{~cm}$ away from the liquid level of the oil sample and lasted for 30 $\min$.

\section{(2) Chromatography condition}

The HP 6980 GC was equipped with DB-WAX GC column $(60 \mathrm{~m} \times 0.25 \mathrm{~mm} \times 0.25 \mu \mathrm{m})$ purchased from Shanghai. Helium was used as a carrier gas at a constant flow rate of $0.35 \mathrm{~mL} / \mathrm{min}$. The initial temperature of $\mathrm{GC}$ was $40{ }^{\circ} \mathrm{C}$ and hold $3 \mathrm{~min}$, then heated to $220^{\circ} \mathrm{C}$ at a rate of $4{ }^{\circ} \mathrm{C} /$ $\mathrm{min}$, followed by the final hold of $10 \mathrm{~min}$. The injection port temperature was $250{ }^{\circ} \mathrm{C}$.

\section{(3) Mass spectrometry condition}

An electron impact ionization was applied at $70 \mathrm{eV}$ with a full scan mass range of $\mathrm{m} / z$ 32-500. The temperature of ion source was $230{ }^{\circ} \mathrm{C}$ and the temperature of interface was $250{ }^{\circ} \mathrm{C}$.

\section{Data analysis}

All the measurements were carried out in triplicate and Agilent MS workstations was used to obtain and analyze GC-MS data. MATLAB R2009b software (The Mathworks Inc., Natick, USA) was used to display data, carry out PCA and LDA.

\section{RESULTS AND DISCUSSION}

\section{Total ion current analysis}

Four different grades of rapeseed oils were analyzed by SPME-GC-MS technology and the average total ion current chromatogram of each grade of sample group were calculated and shown in Fig. 1. As shown in Fig. 1, the numbers of VOCs produced by the first and second grade rapeseed oil samples were significantly less than these of the third and fourth grade samples. Compared with other grade samples, there were some characteristic compounds with higher concentration in the first grade rapeseed oil (seen the characteristic peak indicated by the arrow) and the similar phenomena was observed in the third grade oil samples. As shown in the area marked by dotted rectangle box, new substances were generated and original substances disappeared in the process of improving the quality grade of rapeseed oil. With the improvement of oil grade, the amount of original flavor compounds in rapeseed oil decreased, however, some common components were retained. NIST 


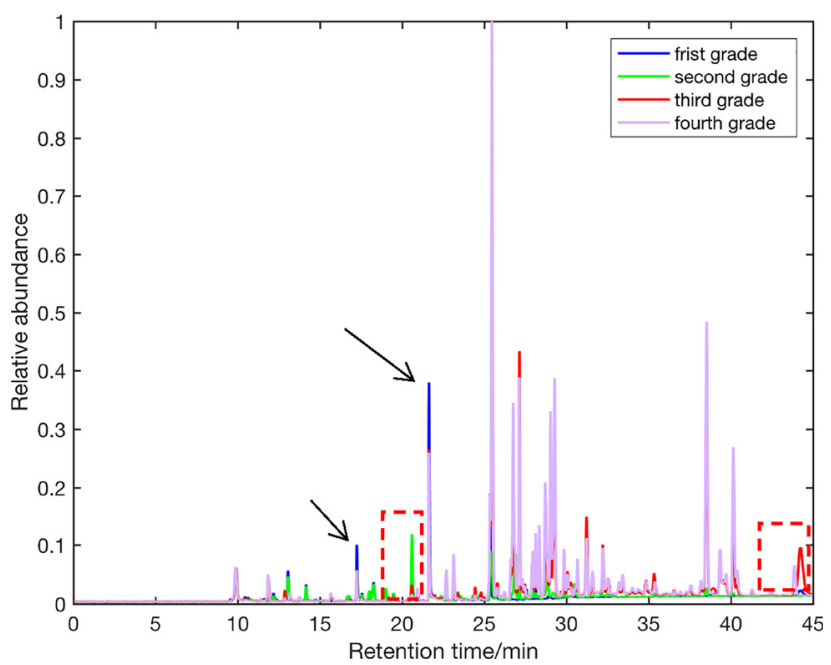

Fig. 1. Total ion current chromatograms of four grades of rapeseed oil

2014 library was used to search and identify flavor substances and only compounds with matching degree greater than or equal to 75 were screened and retained. The relative content of each substance was calculated using the area normalization method. The statistical results were shown in Table 1.

\section{VOCs composition and analysis}

As can be seen from Table 1, a total of 55 volatile flavor substances were identified in four different grades of rapeseed oil samples, including 13 aldehydes, 10 alkanes, 6 nitriles, 5 alcohols, and a small amount of other substances, like esters, pyrazine, furan and pyrrole. The results showed that the number of flavor compounds identified in the first, second, third and fourth grade rapeseed oil samples were 9, 23, 24 and 30 respectively. Small molecular alkanes were the main volatile organic compounds in the first rapeseed oil samples, which accounted for $79.65 \%$ of the total peak area of volatile compounds, and 2,4-dimethylheptane had the highest concentration. The main volatile components in the second grade rapeseed oil samples were aldehydes and alcohols, which accounted for 23.33 and $29.00 \%$ of the total peak area respectively. The third grade rapeseed oil samples mainly contained aldehydes and nitriles, which accounted for 23.87 and $40.51 \%$ respectively. In addition, pyrazine, furan, pyrrole and other VOCs were also found in rapeseed oil. Compared with other three grades of rapeseed oils, the fourth grade oil samples had more characteristic volatile components, which even accounted for about half of the identified substances in number. It was the difference in the amount and content of these volatile organic components that made it possible to distinguish different grades of rapeseed oil.

Aldehydes. Aldehydes in rapeseed oil mainly came from the decomposition products of peroxides formed by oxidation of unsaturated fatty acids, which played an important role in the overall flavor of vegetable oil [18]. The overall reaction process was shown in Fig. 2. It can be seen that the first grade rapeseed oils only contained heptaldehyde, which had fruit flavor characteristics. The number of aldehydes in the second, third and fourth grade rapeseed oils were 5, 7 and 5 respectively, and the second grade rapeseed oil had unique aldehyde substances, such as valeraldehyde, trans-2-hexenal, octanal and nonanal. The former two substances had green leaf and fruit aromas, hexenal and octyl aldehyde had unpleasant odors of citrus, grass or fat, among which nonanal mainly originated from the oxidation of oleic acid and had the aroma of rose and orange [19]. The aldehydes of third grade rapeseed oil included 2-butenal, 3-furfural and 2pyrrolidine formaldehyde, which had pungent odor. The fourth grade samples only contained two special aldehydes (isobutyraldehyde and furfural) and both of them had special pungent odor. The main reason for the formation of trans-2-hexenal and 2-butenal may be that the existence of double bonds at both ends of hydroperoxides lead to the formation of aldehydes, while other aldehydes mainly come from the breakage of single bond at both ends of hydroperoxide [20, 21] (seen area marked by letter A and B in Fig. 2). Refining technology could promote vegetable oil oxidation process in the refining progress. Therefore, the contents of aldehydes (like hexanal, trans-2-hexenal, octanal, nonanal and 3-furfural) were increased in degumming, deacidification and decolorization process [11]. With the refining process going on, the type and quantity of these substances were significantly different from the flavor from main components of crude rapeseed oil. And after deodorization process, these flavor substances were removed by saturated water vapor and finally only heptaldehyde was retained in the first grade rapeseed oil.

Alcohols. Small alcohols also significantly contribute to the flavor of rapeseed oils. As with aldehydes, alcohols mainly came from a series of decomposition of mono-hydroperoxide produced by the air oxidation of rapeseed oil or enzymatic reactions [22]. As shown in Table 1, only 2chloroethanol was detected in the first grade rapeseed oil, three alcohols were found in the second grade oil, which were 1-pentanol, 1-hexanol and 2-methyl-6-heptane-1-ol, respectively. The two former compounds had grass smell and were the characteristic flavors of rapeseed oil. On the other hand, only furfural alcohol was found in the third and fourth grade rapeseed oil, which made the rapeseed oil present barbecue flavor and was a typical Maillard reaction product.

Pyrazines. Pyrazines were mainly derived from Maillard reaction during the preparation of rapeseed oil. These substances had the characteristics of low threshold and good divergence, and played an important role in the formation of oil flavor [23]. It can be seen from Table 1 that there were 5 pyrazines in the fourth grade rapeseed oil. Compared with other grades of oil samples, the types and contents of pyrazines in the fourth grade rapeseed oil were higher and were significantly reduced with the deepening of refining degree. 
Table 1. Volatile composition and relative contents of four grades of rapeseed oil samples $(n=3)$

\begin{tabular}{|c|c|c|c|c|c|c|}
\hline \multirow[b]{2}{*}{ Index } & \multirow[b]{2}{*}{ Retention time/min } & \multirow[b]{2}{*}{ Compound } & \multicolumn{4}{|c|}{ Relative content calculated by area normalization/\% } \\
\hline & & & Grade 1 & Grade 2 & Grade 3 & Grade 4 \\
\hline 1 & 10.44 & 2-Methylpentane & 0.80 & - & - & - \\
\hline 2 & 11.85 & Carbon disulfide & - & - & - & 0.71 \\
\hline 3 & 12.18 & 2-Methylheptane & 1.58 & 0.86 & - & - \\
\hline 4 & 12.87 & Octane & 0.74 & 1.30 & 0.33 & 0.08 \\
\hline 5 & 13.06 & 2,4-Dimethylheptane & 4.48 & 3.35 & - & - \\
\hline 6 & 13.18 & Isobutyraldehyde & - & - & - & 0.27 \\
\hline 7 & 14.16 & 4-Methyloctane & 2.27 & 1.81 & - & - \\
\hline 8 & 15.66 & 2-Methylbutyraldehyde & - & - & 0.19 & 0.30 \\
\hline 9 & 17.56 & Pentanal & - & 0.86 & - & - \\
\hline 10 & 17.99 & Decane & 1.39 & 1.23 & - & - \\
\hline 11 & 19.00 & 4,7-Dimethyl undecane & - & 1.25 & - & - \\
\hline 12 & 19.15 & 4,6-Dimethyl undecane & 0.70 & 0.46 & - & - \\
\hline 13 & 19.44 & 2-Butyraldehyde & - & - & 0.16 & - \\
\hline 14 & 20.58 & Hexanal & - & 8.72 & 0.50 & 0.22 \\
\hline 15 & 20.93 & 2-Butenenitrile & - & - & 0.39 & 0.38 \\
\hline 16 & 22.36 & m-Xylene & - & 1.14 & - & - \\
\hline 17 & 22.69 & 3-Butenenitrile & - & - & 0.78 & 0.80 \\
\hline 18 & 23.14 & Crotononitrile & - & - & 0.77 & - \\
\hline 19 & 23.38 & Heptaldehyde & 1.22 & - & 0.25 & - \\
\hline 20 & 23.54 & Dodecane & - & 0.63 & - & - \\
\hline 21 & 24.31 & Trans-2-hexenal & - & 0.77 & - & - \\
\hline 22 & 24.44 & 2-Pentylfuran & - & - & 0.34 & 0.13 \\
\hline 23 & 24.73 & 1-Pentanol & - & 0.92 & - & - \\
\hline 24 & 25.46 & 2-Methyl-3-butenenitrile & - & 6.21 & 15.70 & 15.22 \\
\hline 25 & 25.78 & 1-Butyne & - & - & - & 0.52 \\
\hline 26 & 25.81 & Octanal & - & 1.67 & - & - \\
\hline 27 & 26.61 & 2,4-Pentadienenitrile & - & - & 0.75 & 0.88 \\
\hline 28 & 26.75 & 2,5-Dimethyl pyrazine & - & - & 3.52 & 5.85 \\
\hline 29 & 26.89 & 1-Hexanol & - & 7.53 & - & - \\
\hline 30 & 26.99 & Ethylpyrazine & - & - & - & 0.193 \\
\hline 31 & 27.56 & 2-Chloroethanol & 1.84 & - & - & - \\
\hline 32 & 27.92 & 1-Nonanal & - & 1.72 & - & - \\
\hline 33 & 27.95 & 2-Ethyl-6-methylpyrazine & - & - & - & 1.47 \\
\hline 34 & 28.13 & 2-Ethyl-5-methylpyrazine & - & - & 1.35 & 1.57 \\
\hline 35 & 28.35 & Trimethyl-pyrazine & - & - & - & 1.73 \\
\hline 36 & 28.73 & Acetic acid & - & - & 3.40 & 4.35 \\
\hline 37 & 28.87 & 2,4-Mimethyl-2,6-heptadien-1-ol & - & 2.60 & - & - \\
\hline 38 & 29.02 & 2,5-Dimethyl-3-ethylpyrazine & - & - & 2.07 & 4.31 \\
\hline 39 & 29.17 & 2,4-Dimethylthiazole & - & 1.10 & - & - \\
\hline 40 & 29.27 & Furfural & - & - & - & 6.99 \\
\hline 41 & 29.27 & 3-Furaldehyde & - & - & 6.56 & - \\
\hline 42 & 30.06 & 2-Acetylfuran & - & - & 0.87 & 0.76 \\
\hline 43 & 30.49 & Octyl formate & - & 2.17 & - & - \\
\hline 44 & 31.22 & 5-Methyl furfural & - & - & 2.73 & 1.78 \\
\hline 45 & 31.58 & Dimethyl sulfoxide & - & - & 0.83 & 1.09 \\
\hline 46 & 32.21 & Furfuryl alcohol & - & - & 2.05 & 1.75 \\
\hline 47 & 32.29 & 15-Crown-5 & - & 0.37 & - & - \\
\hline 48 & 32.51 & Gamma Butyrolactone & - & - & - & 1.00 \\
\hline 49 & 33.41 & 2-Acetyl-3-methylpyrazine & - & - & - & 0.98 \\
\hline 50 & 37.57 & Benzeneacetonitrile & - & - & - & 0.61 \\
\hline 51 & 38.17 & 2-Acetyl pyrrole & - & - & 0.44 & 0.57 \\
\hline 52 & 39.53 & Pyrrole-2-carboxaldehyde & - & - & 0.70 & - \\
\hline 53 & 40.14 & 3-Phenylpropionitrile & - & - & 2.79 & 5.02 \\
\hline 54 & 40.38 & 2-Pyrrolidinone & - & - & 0.54 & 1.01 \\
\hline 55 & 43.86 & 4-Hydroxy-3-methoxystyrene & - & - & - & 1.20 \\
\hline
\end{tabular}

Note: “-” indicates that it was not detected. 


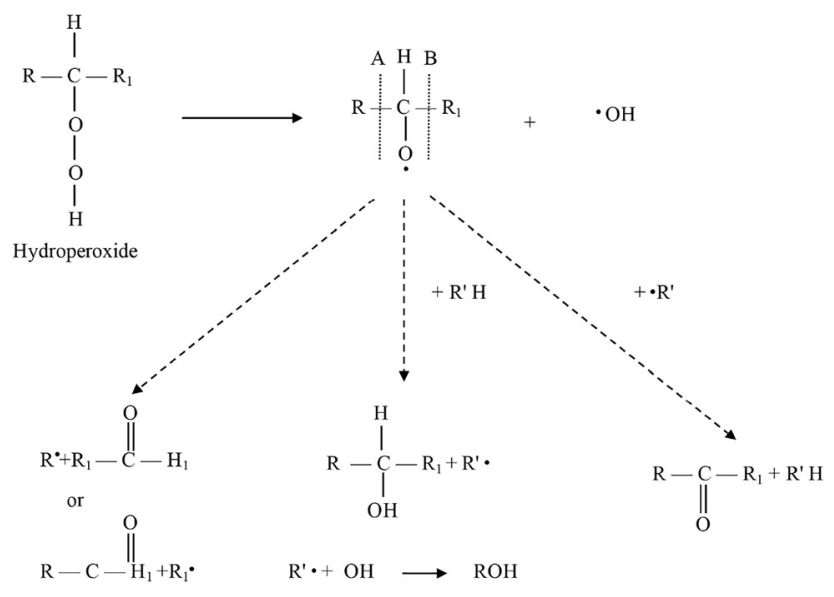

Fig. 2. Decomposition process of hydroperoxides

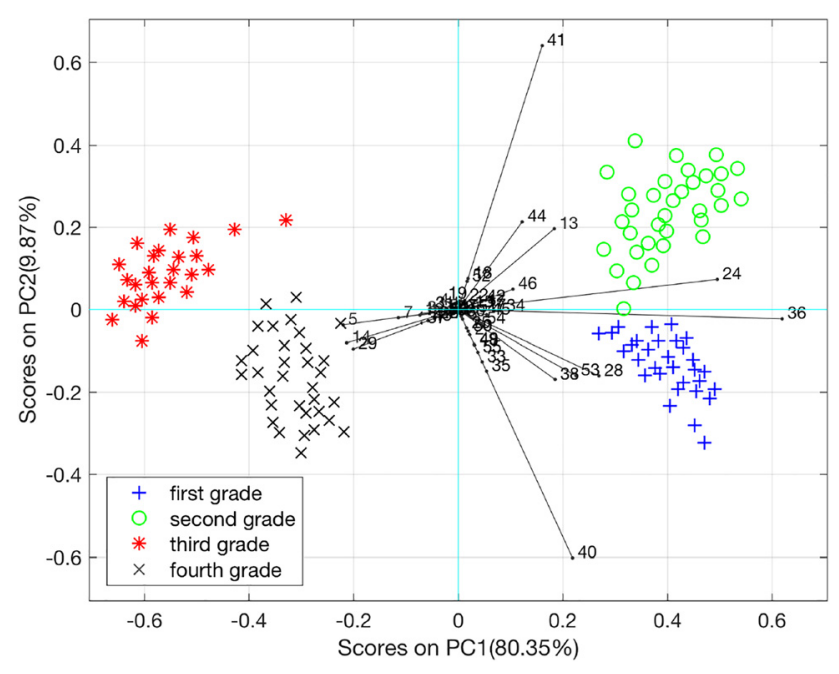

Fig. 3. Principal component scores and loading distribution plot

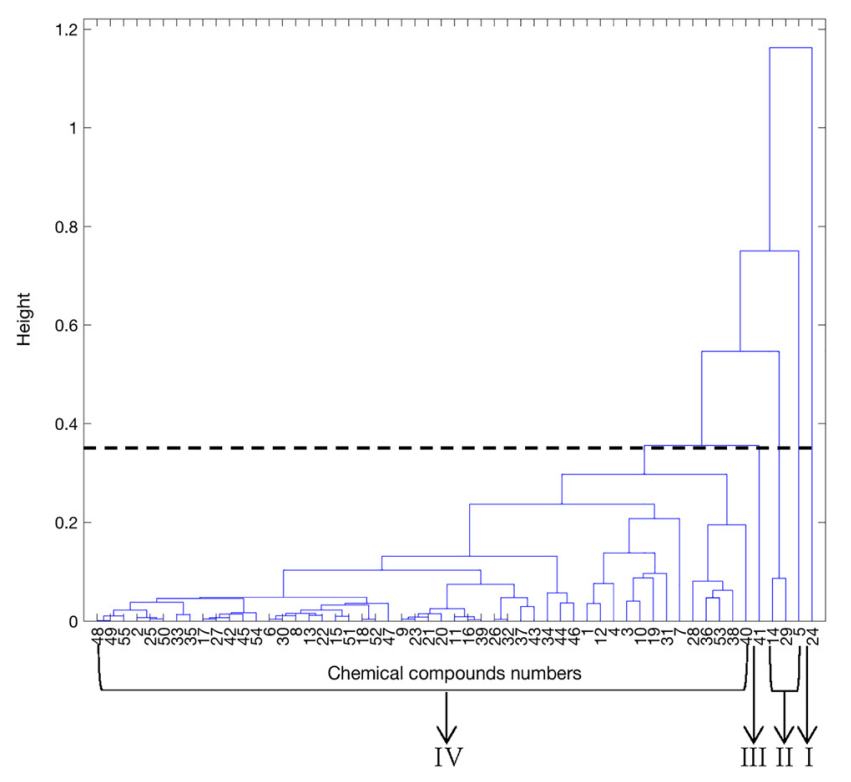

Fig. 4. Cluster analysis of different grade rapeseed oil samples
No pyrazines were found in the first and second grade rapeseed oil samples. 2,5-dimethylpyrazine, 2-ethyl-5methylpyrazine, 2,3-methyl-2-methyl-pyrazine and 2,5dimethylpyrazine were detected in the fourth grade rapeseed oil, which had significant nutty and baking flavor. After degumming process, these substances were distilled and volatilized in the process of oil dehydration, and were gradually removed.

Nitriles. Nitriles mainly come from the degradation of glucosinolates. It is known that glucosinolates are sulfurcontaining secondary metabolites, which widely exist in cruciferae plant. The degradation process of glucosinolates is very complex and the main degradation products are nitriles, nitrogen compounds and sulfur nitrogen compounds, which are the important characteristic flavor compounds in the rapeseed oil and are also the main reason for pungent taste of rapeseed oil [24]. From Table 1, there were 6 kinds of nitriles in the rapeseed oil samples and these substances mainly existed in the third and fourth grade rapeseed oil samples. The second grade rapeseed oil only contained 2methyl-3-butene nitrile, while the first grade oil samples did not contain any nitriles. With the deepening of refining degree, these corresponding substances were gradually decreased, even completely removed. The main reason for this phenomenon was that the degradation products of glucosinolates were destroyed, transferred and preserved in the cake after degumming, deacidification, decolorization and deodorization processes, which made the types and relative content of these substances decrease greatly.

\section{Multivariate analysis}

Chemometrics is a subject that links the measured values of chemical system with the characteristic variables of other measurement system via statistical or mathematical methods. It can be used to optimize experimental methods in order to obtain better measurement data or obtain useful information from measurement data. As can be seen from the previous section, it was very easy to view that there were obvious volatile flavor differences between rapeseed oils with different grades. However, these analyses only relied on the preliminary judgment of qualitative substances, which was difficult to realize digital expression and could not meet the practical application. Therefore, chemometric tools were needed to conduct further analysis, which had been proved to be useful and powerful for data analysis.

These relative contents of 55 identified VOCs were used as characteristic variables, and the content of some undetected VOCs was set 0 by default. These variables were arranged to form a $124 \times 55$ matrix and PCA algorithm was applied to process and analyze the formed matrix. According to the cumulative contribution rate, the obtained principal component scores were sorted from high to low and the first two principal component scores were selected to draw the scatter diagram of different grade oil samples. The results of PCA were shown in Fig. 3. As can be seen, PC1 and PC2 explained $90.22 \%$ of the original information, which meant that the first two principal components could 

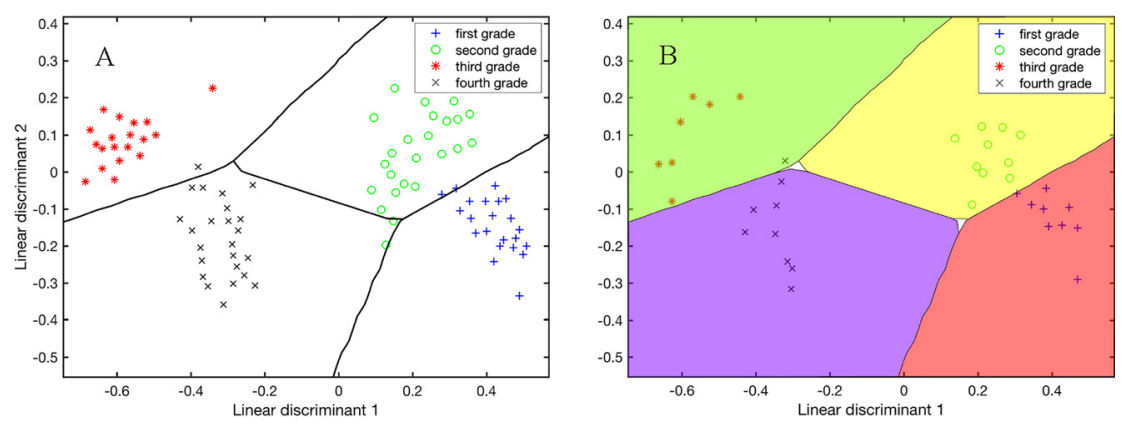

Fig. 5. LDA classification results of the model from training set (A) and testing set (B)

present the most information of the data set. And plotting PC1 and PC2 resulted in slight clustering of the four groups of rapeseed oils. At the same time, the loading matrix (see the black line and corresponding identification substance markers in Fig. 3) was also visualized. Combined with the plot of PCA analysis, the cluster analysis based on Euclidean distance (see Fig. 4) was further applied to analyze the correlation of these features and the feasibility of discriminant analysis. As shown in Fig. 3, different grades of rapeseed oil samples were distributed in the four corners of the PCA diagram. Compared with other VOCs, the feature variables of characteristic substances (markers 5, 14, 29, 13, $24,28,36,38,40,42,44,53$ ) were more important compounds, because their positions were further apart from the origin of coordinates. Some characteristic variables that could be used to distinguish first grade and second grade rapeseed oil samples (e.g. markers 24 and 36) were 2methyl-3-butenenitrile and acetic acid. Although there were still a few of characteristic compounds between third and fourth grade oil samples (e.g. markers 5, 14 and 29) that could be used to make a distinction boundary, these feature variables were correlative and the discrimination effect might not be ideal from the observer's point of view. As shown in Fig. 4, 55 characteristic feature variables could be divided into four categories at the Euclidean distance of 0.38 (see black dotted line). The first category only contained 2methyl-3-butyronitrile (marker 24), the second category included 2,4-dimethylheptane, hexanal and 1-hexanol (markers 5, 29, 14) and the latter two compounds had the aroma of green grass or fruit, which were all the typical characteristic flavor compounds of rapeseed oil. The third category only contained 3-furaldehyde (marker 41), which had a pungent odor and might be related to the rich baking flavor of rapeseed oil. The fourth category included furfural, 2,5-dimethyl-3-ethylpyrazine, 3-phenylpropionitrile, 2,5dimethyl pyrazine and many other less important compounds (e.g. markers 40, 38, 53 and 28). In conclusion, the results of the clustering analysis were consistent with the results of PCA, which indicated that the rapeseed oil samples could be divided into four groups and significant correlations were observed among the feature variables in each group. However, it should be noted that there were relatively few feature variables between third grade and fourth grade oil samples, and these characteristic variables were close to the coordinate origin, which meant that these variables were of low importance in determining quality of the oil samples. On the other hand, it was also important to realize that the cluster boundary between the first grade and the second grade rapeseed oil samples was not clear, which meant it was not easy to distinguish grade 1 and grade 2 rapeseed oils. Therefore, it was necessary to further use chemometric methods to construct a non-linear model for pattern recognition.

LDA algorithm is a simple technique to generate nonlinear boundaries between classes. LDA algorithm attempts to find a linear combination of features that characterizes or separates two or more classes of objects or events. The scoring matrices of the first two principal components were selected as input variables and an LDA classifier was applied to find out the percentage of correct classification of the chemometric model. Before building the model, $70 \%$ of the samples were randomly selected to form the calibration set for training model and the rest $30 \%$ samples were used to evaluate the model performance. The accuracy of classification was used as an indicator of the performance of a classifier. The classification results were shown in Fig. 5. As can be observed, the accuracy of the training set was 97.20 and $94.59 \%$ prediction was obtained using the generated model with only 2 samples misclassified. In general, the whole sample set obtained a good classification. Therefore, the method developed in this study verifies that different grades of rapeseed oils can be distinguished successfully based on flavor and chemometrics.

\section{CONCLUSIONS}

In this paper, GC-MS has been proposed to analyze the VOCs differences of the rapeseed oil with different grades. 124 oil samples were analyzed, 55 volatile flavor substances were identified and the relative contents of these flavor compounds were analyzed by PCA algorithm for feature extraction. Finally, a LDA discriminant model was constructed and the prediction accuracy of sample set showed a good classification of different grade rapeseed oils.

There are significant differences in type, content and quantity of VOCs in rapeseed oil samples of different grades, which are closely related to refining process. The relationship between the refining process of rapeseed oil and the changes of rapeseed oil VOCs could provide a theoretical 
basis for the feasibility of rapid identification of vegetable oil grade by GC-MS. Therefore, GC-MS can be seen as a powerful authentication method in combination with chemometrics, which can also be referenced and applied in other areas.

Conflict of interest: The authors all have declared no conflict of interest.

\section{REFERENCES}

1. Petersen, K. D.; Kleeberg, K. K.; Jahreis, G.; Busch-Stockfisch, M.; Fritsche, J. Eur. J. Lipid Sci. Technol. 2012, 114, 1193-203.

2. Sghaier, L.; Cordella, C. B.; Rutledge, D. N.; Watiez, M.; Breton, S.; Kopczuk, A.; Sassiat, P.; Thiebaut, D.; Vial, J. Chromatographia 2015, 78, 805-17.

3. Matheis, K.; Granvogl, M. Eur. Food Res. Technol. 2016, 242, 1565-75.

4. Azarbad, M. H.; Jeleń, H. Food Anal. Methods 2015, 8, 1727-33.

5. Matheis, K.; Granvogl, M. J. Agr. Food Chem. 2016, 64, 8168-78.

6. Chen, T.; Qi, X.; Lu, D.; Chen, B. J. Food Measurement Charact. 2019, 1-7.

7. Zhang, Y.; Wu, G.; Chang, C.; Lv, Y.; Lai, W.; Zhang, H.; Wang, X.; Jin, Q. Eur. J. Lipid Sci. Technol. 2020, 122, 1900332.

8. Peng, D.; Bi, Y.; Ren, X.; Yang, G.; Sun, S.; Wang, X. Food Chem. 2015, 188, 415-21.

9. Tian, L.; Zeng, Y.; Zheng, X.; Chiu, Y.; Liu, T. Food Anal. Methods 2019, 12, 2282-92.
10. Zhang, L.; Li, P.; Sun, X.; Wang, X.; Xu, B.; Wang, X.; Ma, F.; Zhang, Q.; Ding, X. J. Agr. Food Chem. 2014, 62, 8745-51.

11. Gracka, A.; Jeleń, H. H.; Majcher, M.; Siger, A.; Kaczmarek, A. J. Chromatogr. A. 2016, 1428, 292-304.

12. Xu, L.; Li, X.; Huang, J.; Gao, P.; Jin, Q.; Wang, X. Eur. J. Lipid Sci. Technol. 2019, 121, 1800260.

13. Dạbrowski, G.; Skrajda, M.; Tańska, M.; Roszkowska, B. J. Exp. Agr. Int. 2016, 1-12.

14. Petersen, K. D.; Kleeberg, K. K.; Jahreis, G.; Fritsche, J. Int. J. Food Sci. Nutr. 2012, 63, 160-9.

15. Sghaier, L.; Vial, J.; Sassiat, P.; Thiebaut, D.; Watiez, M.; Breton, S.; Rutledge, D. N.; Cordella, C. B. Eur. J. Lipid Sci. Technol. 2016, 118, 906-18.

16. Sghaier, L.; Cordella, C. B.; Rutledge, D. N.; Watiez, M.; Breton, S.; Sassiat, P.; Thiebaut, D.; Vial, J. J. Sep. Sci. 2016, 39, 1675-83.

17. Zhou, Q.; Yang, M.; Huang, F.; Zheng, C.; Deng, Q. J. Food Sci. 2013, 78, C961-70.

18. Ren, X.; Wang, L.; Xu, B.; Wei, B.; Liu, Y.; Zhou, C.; Ma, H.; Wang, Z. Drying Technol. 2019, 37, 397-408.

19. McDowell, D.; Elliott, C. T.; Koidis, A. Eur. J. Lipid Sci. Technol. 2017, 119, 1600357.

20. Yang, M.; Liu, C.; Zhou, Q.; Zheng, C.; Huang, F. Chin. J. Oil Crop Sci. 2010, 32, 551-7.

21. Yuzhen, L.; Changwen, D.; Yanqiu, S.; Jianmin, Z. J. Food Sci. Eng. 2014, 4, 244-9.

22. Wu, L.; Xu, L.; Xie, J.; Xia, X.; Wang, H. China Oils Fats 2012, 11.

23. Qinzhu, Z.; Yan-ling, C.; Dong-xiao, S.; Tian, B.; Yang, Y.; Shan, H. Lwt. 2018, 97, 573-80.

24. Zhou, Q.; Tang, H.; Jia, X.; Zheng, C.; Huang, F.; Zhang, M. Int. J. Food Prop. 2018, 21, 2296-308. 